\title{
Reconstruction of orbital floor defects assisted by transantral endoscopy
}

\author{
Lucas Borin Moura $^{1}$ - Marisa Aparecida Cabrini Gabrielli ${ }^{1}$. \\ Mario Francisco Real Gabrielli ${ }^{1}$ • Valfrido Antonio Pereira Filho ${ }^{1}$
}

Received: 2 May 2016 / Accepted: 22 September 2016/Published online: 14 October 2016

(C) Springer-Verlag Berlin Heidelberg 2016

\begin{abstract}
Purpose The goal of orbital reconstruction is to restore anatomy, volume, and function. In extensive orbital floor defects, the visualization of the posterior area is limited through inferior eyelid incisions. The use of endoscope may improve the treatment; however, it is a high-sensitivity technique. The aim of this case series is to describe the combination of inferior eyelid incision with transantral endoscopy for treatment of extensive orbital floor defects.

Methods Three patients were submitted to orbital reconstruction, and the postoperative CT scans were evaluated to analyze the orbital volume and anteroposterior globe position. Surgical treatment was performed using subciliary inferior palpebral approach to explore the orbital floor and placement of the titanium mesh and an intraoral antrostomy for endoscopy to magnification of the surgical field and adaptation of the mesh. Results Postoperative CT scan analysis shows that all treatments restored orbital volume and globe position without compression or damage of the optical nerve.
\end{abstract}

Lucas Borin Moura

lucasbmoura@gmail.com

Marisa Aparecida Cabrini Gabrielli

macg@ foar.unesp.br

Mario Francisco Real Gabrielli

mfrg@foar.unesp.br

Valfrido Antonio Pereira Filho

dinho@foar.unesp.br

1 Department of Diagnosis and Surgery, Division of Oral and Maxillofacial Surgery, Dental School at Araraquara, Unesp, Rua: Humaitá, Araraquara, SP 1680, Brazil
Conclusion The use of endoscope allowed the precise visualization of the posterior region of the orbit and adaptation of the titanium mesh.

Keywords Blowout fracture $\cdot$ Endoscopic repair $\cdot$ Orbital floor · Tomography

\section{Introduction}

Orbital fractures represent more than $40 \%$ of all midface fractures. The most prevalent regions are the orbital floor and medial wall [1]. The resulting bone defects may cause a prolapse of orbital content to paranasal sinuses and the entrapment of extraocular muscles [2]. When this occurs, diplopia, enophthalmos, dystopia and ocular movement restriction can be present and surgical treatment is recommended [1-4]. Traditionally, the treatment of orbital floor fractures is performed by inferior eyelid incisions [5]. However, in extensive bone defects, the visualization of posterior orbit and the adaptation of implants can be difficult through those incisions [2, 6].

The use of endoscope in orbital fractures allows a better evaluation and visualization of bone defects and treatment improvement [7]. Small fractures of medial wall and/or orbital floor can be treated only by transnasal or transantral endoscopy without skin incisions [2, 8]. However, in extensive fractures, the reconstruction only with endoscope is difficult and requires a high-sensitivity technique [8]. Alternatively, the combination of inferior eyelid incision and endoscopy is used to treat this kind of fracture [3]. Palpebral approaches provide an easy access for implants and the endoscopy allows the visualization of the posterior region $[5,7]$. The aim of this case series is to describe the reconstruction of extensive orbital floor defects using a combination of palpebral approach and transantral endoscopy and the advantage to use this combination. 
Fig. 1 a Clinical view of the orbital defect. b CT Scan of the orbital fracture

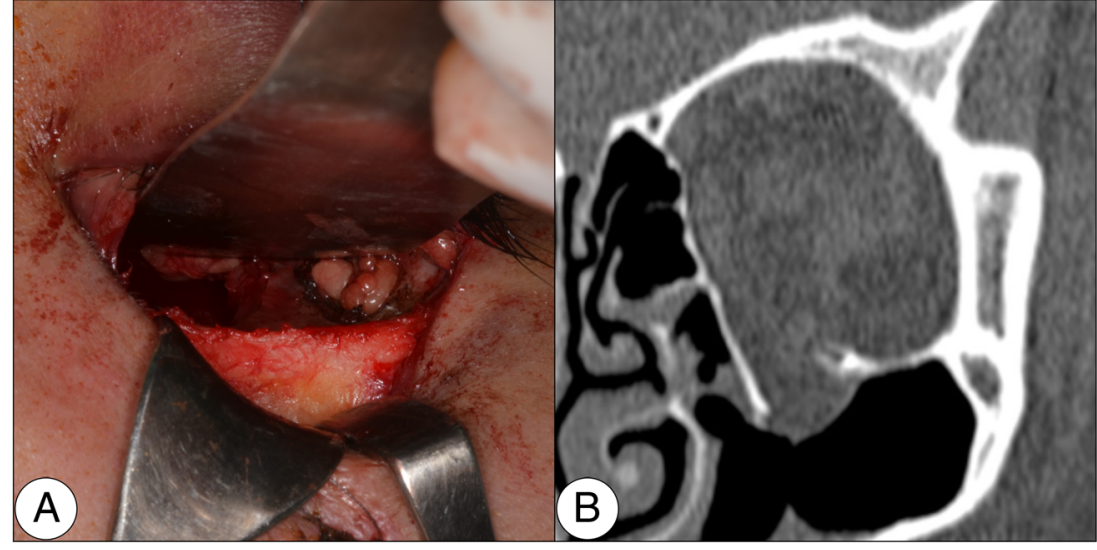

\section{Case series}

Three patients with extensive orbital floor defects were selected to orbital reconstruction using titanium mesh. All patients were treated by inferior eyelid incision and intraoral antrostomy with transantral endoscopy. The patients were placed in reverse Trendelenburg position to facilitate the use of endoscope. The subciliary approach was chosen allowing adequate exposure of the orbital walls (Fig. 1a, b). Simultaneously, through an intraoral incision, a bone window ( $2 \mathrm{~cm}$ width $\times 1 \mathrm{~cm}$ height) was performed in the anterior wall of maxillary sinus, observing a distance of $5 \mathrm{~mm}$ from the apex of the teeth and infraorbital foramen. A 30-degree endoscope was used to explore the maxillary sinus, to remove the sinus mucosa around the fracture and verify defect extension and orbital tissue prolapse. The titanium mesh was placed through palpebral approach. The implant adaptation and absence of entrapment of extraocular muscles were verified using transantral endoscopy (Fig. 2).

A volumetric evaluation of postoperative $\mathrm{CT}$ scans was realized to compare reconstructed and healthy orbits. The DICOM files were imported into software OnDemand 3D 1.0.7.0295 (Cybermed, Seoul, Korea) to make tridimensional volumes and axial, coronal, and sagittal slices. The

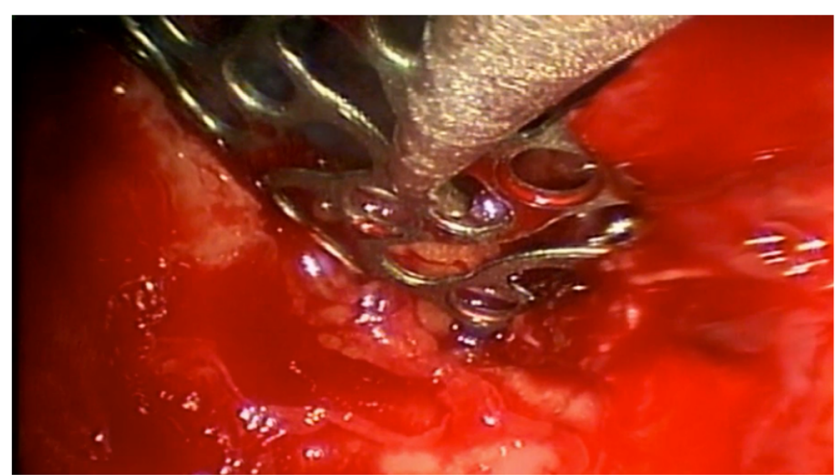

Fig. 2 Endoscopic view of titanium mesh positioning and bone defect extension volumes were reoriented to standardize the head position of all patients. Continuous images with $1-\mathrm{mm}$ thickness were obtained from coronal slices. The initial image was the first slice that showed all orbital rims and the final image was the end of orbital cone. Based on Cavalieri's principle, the sum of areas of the images results in a volume. Differences between orbits up to $8 \%$ are considered anatomical [9].

From the axial slice in the center of the orbit, the anteroposterior position of the eyeball was evaluated. On this image was measured the distance from the posterior region of the central section of the eyeball to a line formed between the zygomatic regions bilaterally [10]. Differences up to $2 \mathrm{~mm}$ are considered anatomically normal [10]. Table 1 shows defects classification, orbital volumes, and difference between the anteroposterior position of eyeballs.

\section{Patient \#1}

A 28-year-old healthy woman suffered car accident 17 days before consult. Physical examination showed a lower eyelid scar, upper eyelid ptosis, enophthalmos, ocular movement restriction, and diplopia in the left eye. CT scan demonstrated left orbital floor fracture with extension to posterior region and involvement of inferior orbital fissure, size more than $2 \mathrm{~cm}^{2}$, a category IV defect [11]. Using the described technique, a titanium mesh was placed to reconstruct the orbit. In the early postoperative period was verified excessive ocular scleral exposure due to scar retraction. The treatment was local physiotherapy. In a month follow-up, there was a resolution of clinical complaints. Volumetric analysis and the eyeball position are according to anatomical limits.

\section{Patient \#2}

A 19-year-old healthy woman was attended in an emergency hospital due to car accident. Physical examination 
Table 1 Patient and orbital characteristics, orbital volume, and difference between the orbits

\begin{tabular}{lllll}
\hline Patient & \multicolumn{2}{l}{ Orbital volume $\left(\mathrm{cm}^{3}\right)$} & Difference $(\%)$ & $\begin{array}{l}\text { Difference of anteroposterior } \\
\text { globe position }(\mathrm{mm})\end{array}$ \\
\cline { 2 - 3 } & Healthy orbit & Reconstructed orbit & & 1.2 \\
\hline Patient \#1 & 15.309 & 15.318 & 0.06 & 1.1 \\
Patient \#2 & 13.677 & 14.503 & 6.04 & -1.9 \\
Patient \#3 & 13.309 & 12.740 & -4.28 & \\
\hline
\end{tabular}

showed diplopia and ocular movement restriction to up and down. In CT scan was observed a category III [11] defect of the right orbital floor with involvement of inferior orbital fissure. The combination of inferior eyelid incision with transantral endoscopy allowed the signal and symptomatology resolution and restoration of orbital volume and anteroposterior eyeball position.

\section{Patient \#3}

A 33-year-old man was admitted in intensive care unit due to car accident with multiple body fractures. Oral and maxillofacial evaluation was required due to associated facial trauma. In the physical exam, the patient was sedated and unresponsive. A facial asymmetry in the left zygomatic region was observed. CT scan showed zygomatic fracture with extensive orbital defect in the floor and medial wall-category IV [11]. Surgical treatment was performed using the combination of approaches and a supraciliary approach to reduce the sphenozygomatic suture. Postoperative period was uneventful; however, due to systemic injuries the patient died.

\section{Discussion}

Usually, orbital floor fractures are comminuted and these bone defects may extend to the posterior area or medial wall according to trauma intensity $[3,12]$. Inadequate treatment may lead to sequelae such as diplopia, increased orbital volume and enophthalmos, ocular movement restriction due to entrapment of extraocular muscles, infraorbital nerve paresthesia, and blindness [4]. All reported cases had indication of surgical treatment due to presence of orbital defects larger than $2 \mathrm{~cm}^{2}$, enophthalmos, diplopia, and orbital content prolapse.

The goal of orbital reconstruction is to restore anatomy, volume, and function. However, the visualization and adaptation of implants in posterior area defects can be difficult through palpebral approaches [3]. The use of endoscopy allows magnification and visualization of all surgical fields [3]. Trapdoor fractures and small defects can be treated through endoscopic approach without skin incisions, but extensive defects require high technical sensitivity for placement of implants [5, 6]. Therefore, due to the extension of the defects, we use the combination between eyelid incision and transantral endoscopy to optimize the visualization and treatment of the patients.

The maxillary sinus represents an adjacent cavity to the orbital floor and can be used for endoscopic approach [7]. The use of 30-degree endoscope may improve the orientation of surgeon and help to identify anatomical structures such as orbital floor and bone defects, prolapsed tissue, infraorbital nerve, and maxillary sinus ostium [13]. In addition, the endoscope allows better understanding of the characteristics of the fracture and improves the adaptation of the implants [8].

The described combination of approaches shows few limitations or disadvantages. The possibility of obvious scars and complications in eyelid incisions are discussed in literature. However, complications are present in only $5 \%$ of cases and are less common in isolated orbital wall fractures [2]. In this case series was observed a transient excessive exposure of eye sclera. Transantral endoscopy complications are rare and related to their isolated use by inexperienced operator [4]. However, the antrostomy is associated to postoperative paresthesia and is contraindicated for children when the dental germs are present adjacent to a small maxillary sinus [5].

In this case series, the described technique allowed a simplified approach to orbital floor fractures, examination of defects extension, and titanium mesh positioning. In all reconstructions, the orbital volume was restored observing anatomical differences of up to $8 \%$ [9]. The greatest difference between the orbital volumes was verified in patient \#3. This patient had a zygomatic fracture involving the lateral wall which causes an increase of orbital volume. In addition, the increased volume is related to the major difference between the anteroposterior eyeball positions observed in Table 1 . However, all values are within the normal anatomical differences.

In conclusion, the combination of eyelid incision and transantral endoscopy is a simple and easy technique that allows to optimize the visualization and reconstruction of the orbital floor. The postoperative CT scan analysis shows that all treatments restored orbital volume and globe position. 
Acknowledgments The authors thank the funding agency FAPESP (Foundation for Research Support of Sao Paulo) for the support to this article (FAPESP process: 2015/04521-0).

\section{References}

1. Ellis E 3rd (2012) Orbital trauma. Oral Maxillofac Surg Clin North Am 24:629-648. doi:10.1016/j.coms.2012.07.006

2. Farwell DG, Strong EB (2007) Endoscopic repair of orbital floor fractures. Otolaryngol Clin N Am 40:319-328

3. Nahlieli O, Bar-Droma E, Zagury A et al (2007) Endoscopic intraoral plating of orbital floor fractures. J Oral Maxillofac Surg 65:1751-1757

4. Lee DG, Cho KS, Kim HJ, Roh HJ (2012) Cerebrospinal fluid rhinorrhea as a complication of endoscopic endonasal reduction of blowout fractures. J Laryngol Otol 126:942-945. doi:10.1017 /S0022215112001399

5. Kakibuchi M, Fukazawa K, Fukuda K et al (2004) Combination of transconjuntival and endonasal-transantral approach in the repair of blowout fractures involving the orbital floor. Br J Plast Surg 57:37-44

6. Pham AM, Strong EB (2006) Endoscopic management of facial fractures. Curr Opin Otolaryngol Head Neck Surg 14:234-241
7. Schubert W, Jenabzadeh K (2009) Endoscopic approach to maxillofacial trauma. J Craniofac Surg 20:154-156. doi:10.1097/SCS.0 b013e318191cfb8

8. Strong EB, Kim KK, Diaz RC (2004) Endoscopic approach to orbital blowout fracture repair. Otolaryngol Head Neck Surg 131:683-695

9. Parson GS, Mathog RH (1988) Orbital wall and volume relationships. Arch Otolaryngol Head Neck Surg 114:743-747

10. Strianese D, Piscopo R, Elefante A et al (2013) Unilateral proptosis in thyroid eye disease with subsequent contralateral involvement: retrospective follow-up. BMC Ophthalmol 13:1-9. doi:10.1186 /1471-2415-13-21

11. Jaquiéry C, Aeppli C, Cornelius P, Palmowsky A, Kunz C, Hammer B (2007) Reconstruction of orbital wall defects: critical review of 72 patients. Int J Oral Maxillofac Surg 36:193-199

12. Park AH, Diaz JÁ (2008) A different approach to orbital blow out fractures: microscope-assisted reconstruction of the orbital floor. Int J Pediatr Otorhinolaryngol 72:707-710. doi:10.1016/j. ijporl.2008.01.016

13. Moore CC, Bromwich M, Roth K, Matic DB (2008) Endoscopic anatomy of the orbital floor and maxillary sinus. J Craniofac Surg 19:271-276. doi:10.1097/SCS.0b013 e31815ca18b 